\title{
A Scoping Review of Community-Based Geriatric Health Assessment and Screening Tools used in South Asia
}

Sucharita Panigrahi ${ }^{1}$, Trilochan Bhoi ${ }^{1}$, Sanghamitra Pati ${ }^{1}$, Jaya Singh Kshatri ${ }^{1 *}$

${ }^{1}$ ICMR- Regional Medical Research Centre, Chandrasekharpur, Bhubaneswar, Odisha, India *Corresponding author: Dr. Jaya Singh Kshatri Email: jsk.icmr@outlook.com Address: ICMR- Regional Medical Research Centre, Chandrasekharpur, Bhubaneswar, Odisha 751023, India

\section{Abstract}

\section{Background}

Home-based comprehensive assessment and integrated care of the older people could be a key to relieve the pressure on the already overburdened health system. This review summarises evidence on validated community-based geriatric health assessment tools in South Asia.

\section{Methods}

Guided by Arksey and O'Malley's five-stage scoping review framework, a total of 46 studies were included in the scoping review after searching from electronic databases and reference lists using predefined eligibility criteria. Data were extracted on main characteristics of included studies, identified instruments and their psychometric properties of the tools. This review was reported in accordance with PRISMA-ScR guidelines.

\section{Results}

Among the 46 included studies, 10 reported on instruments for medical assessment, 12 on tools for psychological conditions, 13 on tools for functional issues, 2 on social wellbeing, and 9 on tools with multiple domains of health. Most studies included participants from both gender and different social classes. Majority used measurements that were both self-reported or measured by the investigator. whereas only two instruments were designed to be used by clinicians. In the existing 
geriatric health assessment tools, environmental domain was neglected completely, and not a single tool considered in this review covered all 5 domains which influence regular life of elderly.

\section{Conclusion}

There are no validated tools available that can be used for comprehensive geriatric assessment in South Asia. There is a need to develop and validate culturally sensitive tools that can be used for assessing all the geriatric health domains.

Keywords: Geriatric health, Health Assessment tool, Validated tools, Screening tools, 
medRxiv preprint doi: https://doi.org/10.1101/2021.02.19.21252051; this version posted May 22, 2021. The copyright holder for this preprint

(which was not certified by peer review) is the author/funder, who has granted medRxiv a license to display the preprint in perpetuity.

It is made available under a CC-BY 4.0 International license .

\section{Introduction}

An increasingly older population is contributing to a majority of disease burden and subsequently revamping healthcare expenditures globally[1] [2][3]. This burden is more acute in low-income settings where the need for healthcare in older age groups is greater and coverage of health and social security schemes is inadequate[4]. Projections have shown that an overwhelming proportion of global disease burden will be from agerelated disorders in the foreseeable future[5].

In this situation, Comprehensive geriatric assessment (CGA) is recognized as a key intervention to address the health needs of older adults, effectively evaluate comorbid conditions and functional limitation[6][7]. Even though, there is no standardized format for carrying out CGA, using multiple tools to capture comprehensive health status, a consensus on the broad domains that need to be measured[8][6]. Most of the tools available focus on a particular domain of CGA and a majority of these have been validated for use in high-income countries(HIC), where they have been developed[9][10][11]. Concerns on the utility and accuracy of these tools in Low and Middle-income countries (LMIC)remain [12]. Unlike clinical evaluation, CGA involves complex constructs that are influenced strongly by the socio-cultural milieu of the end-users and target population, which are widely diversified across regions, let alone the world. Therefore, tools need contextual adaptation and validation for optimum utility in the targeted settings.

While there still are linguistic and regional differences among regions in South Asia, historically, the subcontinent has had a common sociocultural thread running through it as well as a common administrative architecture, making generalizability of health interventions easier. This scoping review aims to summarize studies that describe validated tools for assessing geriatric health in community settings in South Asia. We believe there is no single tool validated for CGA and there is no structured evidence synthesis conducted to map empirical evidence for tools specifically for this population. So, this review will provide evidence to researchers and practitioners on available validated tools, their psychometric properties, and validity in order to enable them to make an informed choice on which tools to include in community-based CGA. Findings of this review could also aid in identifying scope for updating of 
medRxiv preprint doi: https://doi.org/10.1101/2021.02.19.21252051; this version posted May 22, 2021. The copyright holder for this preprint (which was not certified by peer review) is the author/funder, who has granted medRxiv a license to display the preprint in perpetuity. It is made available under a CC-BY 4.0 International license .

existing tools for the South Asia population as well as identify crucial gaps in the domain of CGA where validated tools are missing.

\section{Methods}

This scoping review was reported in accordance with the Preferred Reporting Items for Systematic Reviews and Meta-Analyses statement for reporting systematic reviews-extension for scoping reviews[13] presented in Appendix-1 and was prospectively registered with Open Science Framework(10.17605/OSF.IO/TFR3H).

We followed the Arksey and O'Malley framework [14] for conducting scoping reviews which uses the following steps -

Step1: Identification of Research Question

Step2: Identification of relevant studies

Step3: Study selection

Step4: Data charting

Step5: Data collation, summarize, and reporting the result,

These steps were further refined by Levac et al.[15].

\section{Identification of Research Question}

The research question addressed by this review was developed based on PCC- Population=Geriatric, Concept $=$ Community based health assessment and screening tools, and Context =South Asia.

R.Q: What are the validated community-based geriatric health assessment and screening tools available in South Asia?

\section{Identification of relevant studies}

We conducted a systematic search of the following electronic databases: MEDLINE, Embase and PsycINFO. We searched articles published in English only without any date restrictions. The pilot searches were carried out with variations of the words "Community-based", "elderly", "Health assessment*" "screening", "Tool*", along with countries and states of south Asia that appeared in Title/ Abstracts. The detailed search string is presented in Appendix 2. Additional relevant papers were identified through reference mining and Google Scholar search using similar keywords.

\section{Study selection}


medRxiv preprint doi: https://doi.org/10.1101/2021.02.19.21252051; this version posted May 22, 2021. The copyright holder for this preprint (which was not certified by peer review) is the author/funder, who has granted medRxiv a license to display the preprint in perpetuity. It is made available under a CC-BY 4.0 International license .

After an extensive search, following de-duplication of electronic articles, all eligible articles were screened by two independent review authors (SP1 \& TB) based on their Title/Abstract. Discrepancies were discussed and resolved. In case of disagreement, a third author (JSK) made the decision. Full texts were retrieved and reviewed for eligibility. We included studies conducted in any of the South Asian countries that evaluated geriatric health assessment tools based on community setting, or any of the subdomains or reported its development process or validation. Institutional tools which can be implemented in the community were also included. We excluded manuscripts that were in form of comments, editorials, letters, Conference or congress papers, abstracts, and reviews. Full-text review followed the same method as Title/abstract screening in case of disagreement. In this study, we have not considered methodological rigor of the included studies.

\section{Charting the data}

Through iterative process, a predefined data extraction sheet was developed to capture all relevant aspects of the included studies. The study characteristics like country of origin, objectives, sample characteristics, setting, sample size, and sampling method were extracted. Detailed description of the health assessment tools and the procedure of its development, composition, validity, reliability, and feasibility of the instruments were extracted from the relevant paper. After completion, the charted tables were examined further within the reviewers to ensure accuracy and consistency. The detailed descriptions of the studies are given in appendix-3.

\section{Collating, Summarising, and reporting the results}

A narrative summary of the results was presented. Tables were used to present specific details of the tools and development process. The results section first described the characteristics of the studies, characteristics of the tool, and its psychometric properties. The charted tools were categorized into five broad domains and further subdomains of CGA as follows[16][17].

a. Medical Assessment

b. Functional Assessment 


\section{c. Social Assessment \\ d. Environment Assessment \\ e. Multiple}

Similarly, we also summarized the most reported outcome measures of validity and reliability such as sensitivity, specificity, positive predictive value (PPV), negative predictive value (NPV), and receiver operating characteristics area under the curve (ROC-AUC). We have grouped all the available tools with psychometric properties in 3 categories to make our results standardized.

1. High sensitivity/specificity: When the tool reported value is ranging from $67 \%-100 \%$.

2. Moderate Sensitivity/Specificity: When the tool reported value is ranging from $34 \%-66 \%$

3. Poor Sensitivity/ Specificity: When the tool reported value is ranging from $0 \%-33 \%$

\section{Results}

We identified 607 records from electronic databases and other sources after de-duplication. 116 were identified for full-text screening and 46 studies were included in the final analysis. The PRISMA flow diagram for the study selection is provided in Figure 1. 
medRxiv preprint doi: https://doi.org/10.1101/2021.02.19.21252051; this version posted May 22, 2021. The copyright holder for this preprint (which was not certified by peer review) is the author/funder, who has granted medRxiv a license to display the preprint in perpetuity.

It is made available under a CC-BY 4.0 International license.
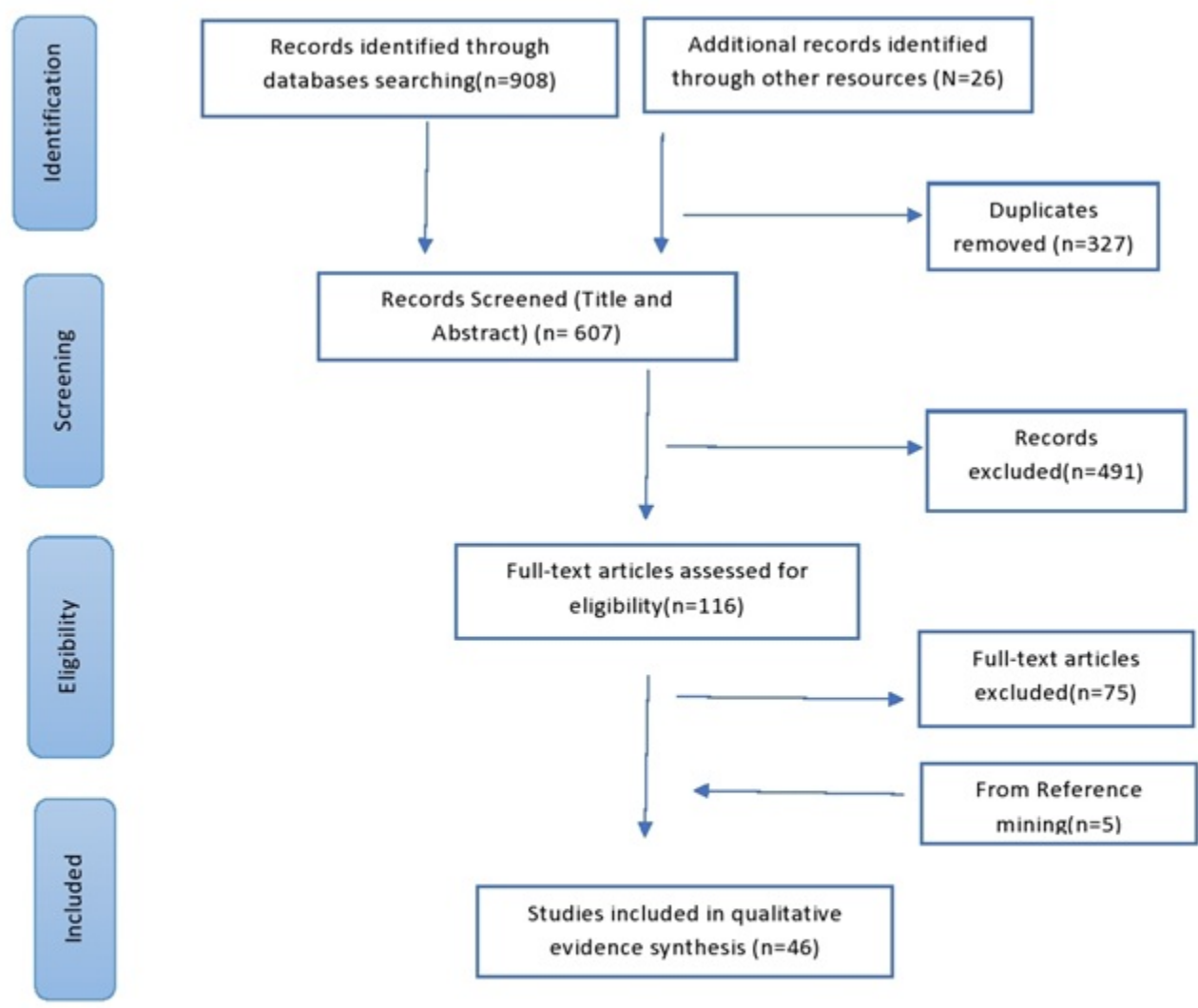

Studies included in qualitative evidence synthesis $(n=46)$

All studies were cross-sectional and aimed to either develop, validate or test tools for assessment of different health parameters. A considerable number were part of larger studies, but a few were multinational in scope, so we are presenting the components that concerned the tool development/validation in South Asia only. All studies included participants from all genders, different age groups and social classes, except Rathnayake-2020, who included only postmenopausal women[18]. The study characteristics are summarized in Table-1 below.

\section{Table-1: - Characteristic of Included studies}




\begin{tabular}{|c|c|c|c|}
\hline Author IYYYY & $\begin{array}{l}\text { Tool } \\
\text { developed/validated/tested }\end{array}$ & $\begin{array}{l}\text { Country of } \\
\text { origin }\end{array}$ & $\begin{array}{l}\text { Study } \\
\text { setting }\end{array}$ \\
\hline Adhikari 2009 & \begin{tabular}{|l|} 
Madras Diabetes Research \\
Foundation (MDRF)- Indian \\
Diabetes Risk Score (IDRS)
\end{tabular} & India & Semi-urban \\
\hline Bhowmik 2015 & $\begin{array}{l}\text { Simple- diabetes risk score } \\
\text { (DRS) }\end{array}$ & Ban'esh & Rural \\
\hline $\begin{array}{l}\text { Chatterjee } \\
2019\end{array}$ & $\begin{array}{l}\text { Integrated care tool (ICT- } \\
\text { BRIEF) }\end{array}$ & India & $\begin{array}{l}\text { Hospital, } \\
\text { community } \\
\text { (Both rural } \\
\text { and urban) } \\
\text { and old } \\
\text { age home }\end{array}$ \\
\hline $\begin{array}{l}\text { Chokkanathan } \\
2013\end{array}$ & $\begin{array}{l}\text { Centre for Epidemiological } \\
\text { Studies - Depression scale } \\
\text { (CES-D) }\end{array}$ & India & Urban \\
\hline Corsi 2012 & $\begin{array}{lr}\text { Environmental } & \text { Profile of a } \\
\text { Community's } & \text { Health } \\
(\text { EPOCH-2) } & \end{array}$ & $\begin{array}{l}\text { Multiple } \\
\text { (India in } \\
\text { south Asia) }\end{array}$ & urban \\
\hline Dandona 2000 & $\begin{array}{l}\text { WHO Quality of Life- for } \\
\text { vision (WHOQOL-vision) }\end{array}$ & India & $\begin{array}{l}\text { Both rural } \\
\text { and urban }\end{array}$ \\
\hline Dasgupta $202 C$ & Alzheimer Questionnaire & India & Urban \\
\hline De Silva 2016 & $\begin{array}{l}\text { Quality of life instrument for } \\
\text { the young elderly in Sri } \\
\text { Lanka (QLI YES) }\end{array}$ & Sri Lanka & $\begin{array}{l}\text { Urban } \\
\text { rural }\end{array}$ \\
\hline Deepthi 2012 & $\begin{array}{lrr}\text { Single } & \text { question } & \text { and } \\
\text { Shortened } & \text { Hearing } \\
\text { Handicap Inventory for } \\
\text { Elderly (HHIE-S) }\end{array}$ & India & Rural \\
\hline Ganguli 1995 & $\begin{array}{|lr|}\text { Hindi Mini-mental } & \text { state } \\
\text { examination (HMSE) }\end{array}$ & India & Rural \\
\hline Husain 2006 & $\begin{array}{lr}\text { Personal } & \text { Health } \\
\text { Questionnaire } & (\mathrm{PHQ}) \text { and } \\
\text { Self } & \text { Reporting } \\
\text { Questionnaire } & \text { (SRQ) }\end{array}$ & & Rural \\
\hline $\begin{array}{l}\text { Jotheeswaran } \\
2016 \\
\end{array}$ & $\begin{array}{l}\text { EASY-care Independence } \\
\text { Scale (EASY Care) }\end{array}$ & India & $\begin{array}{l}\text { Both rura } \\
\text { and urban }\end{array}$ \\
\hline
\end{tabular}




\begin{tabular}{|c|c|c|c|c|}
\hline Khan 2015 & $\mid \begin{array}{l}\text { Questionnaire to Verify } \\
\text { Stroke Free Status (QVSFS) }\end{array}$ & Pakistan & Urban & \\
\hline Marella 2014 & $\begin{array}{|ll|}\text { Rapid assessment of } \\
\text { Disability (RAD) }\end{array}$ & Bangladesh & $\begin{array}{l}\text { Both rura } \\
\text { and urban }\end{array}$ & \\
\hline Misra 2014 & $\begin{array}{l}\text { Global physical activity } \\
\text { questionnaire (GPAQ) }\end{array}$ & India & $\begin{array}{l}\text { Both rura } \\
\text { and urban }\end{array}$ & \\
\hline Moiz 2017 & $\begin{array}{l}\text { Activities-specific balance } \\
\text { confidence in Hindi (ABC-H } \\
\text { scale) }\end{array}$ & India & Urban & $0-\varepsilon$ \\
\hline \begin{tabular}{|l|} 
Nepal, \\
M.,2019
\end{tabular} & Timed up and Go (TUG) test & Nepal & Rural & D-c \\
\hline $\begin{array}{lll}\text { Perera, } & \text { B. } & \text { P. } \\
\text { R.,2020 } & & \\
\end{array}$ & WHO-5 n & Sri Lanka & $\begin{array}{l}\text { Semi- } \\
\text { Urban }\end{array}$ & \\
\hline \begin{tabular}{|l|}
$\begin{array}{l}\text { Poongothai, S., } \\
2009\end{array}$ \\
\end{tabular} & $\begin{array}{ll}\text { Personal } & \text { health } \\
\text { questionnaire (PHQ-9, PHQ- } \\
12 \text { ) }\end{array}$ & India & Urban & \\
\hline $\begin{array}{l}\text { Prince, } \\
\text { M.,2003 }\end{array}$ & $\begin{array}{l}\text { Community Screening } \\
\text { Instrument for Dementia } \\
\text { (CSI 'D'), Informant interview } \\
\text { about daily functioning (IDF), } \\
\text { Consortium to Establish a } \\
\text { Registry of Alzheimer's } \\
\text { Disease (CERAD) } \\
\end{array}$ & (Multiple in & nuni & \\
\hline Qin 2018 & \begin{tabular}{ll|} 
General & Health \\
Questionnaire (GHQ-12)
\end{tabular} & India & $\begin{array}{l}\text { Both rura } \\
\text { and urban }\end{array}$ & \\
\hline $\begin{array}{l}\text { Ramachandran } \\
2005\end{array}$ & DRS- Urban Indians & India & Urban & \\
\hline $\begin{array}{l}\text { Rathnayake } \\
2020\end{array}$ & $\begin{array}{l}\text { Health Promoting Lifestyle } \\
\text { Profile-II (HPLP-II) }\end{array}$ & Sri Lanka & $\begin{array}{l}\text { Both rura } \\
\text { and urban }\end{array}$ & $\begin{array}{l}>40 \\
\text { posi } \\
\text { mer }\end{array}$ \\
\hline Sarkar 2015 & $\begin{array}{l}\text { Geriatric depression scale } \\
\text { (GDS-15) }\end{array}$ & India & Rural & 0 \\
\hline Sherpa 2015 & \begin{tabular}{|l|l|} 
St. George respiratory \\
questionnaire (SGRQ)
\end{tabular} & Nepal & $\begin{array}{l}\text { Both rura } \\
\text { and urban }\end{array}$ & $40-\varepsilon$ \\
\hline Siriwardhana & Instrumental & & & \\
\hline
\end{tabular}




\begin{tabular}{|c|c|c|c|}
\hline 2018 & $\begin{array}{l}\text { Activities of Daily Living } \\
\text { (IADL) }\end{array}$ & Sri Lanka & Rural \\
\hline Stanley 2009 & $\begin{array}{l}\text { Vellore } \\
\text { Instrument for } \\
\text { Patient and } \text { Dementia } \\
\text { (VSID-P and VSID-I) }\end{array}$ & India & $\begin{array}{l}\text { Both rural } \\
\text { and urban }\end{array}$ \\
\hline Stewart 2016 & Short schedule of $10 / 66$ tool & $\begin{array}{l}\text { India (and } \\
\text { others) }\end{array}$ & $\begin{array}{l}\text { Both rural } \\
\text { and urban }\end{array}$ \\
\hline Tausig 2003 & $\begin{array}{l}\text { Modified DSM-III-R Criteria } \\
\text { Checklist }\end{array}$ & Nepal & Rural \\
\hline Vaz, M., & $\begin{array}{ll}\text { Semi-pictorial } & \text { Rural Physical } \\
\text { Activity } & \text { Questionnaire } \\
\text { (RPAQ) } & \\
\end{array}$ & India & Rural \\
\hline Pati 2016 & $\begin{array}{l}\text { Multimorbidity assessment } \\
\text { in primary care (MAQ-PC) }\end{array}$ & tIndia & $\begin{array}{l}\text { Both urban } \\
\text { and rural }\end{array}$ \\
\hline Mohan 2005 & $\begin{array}{l}\text { Indian Diabetes Risk Score } \\
\text { (IDRS) }\end{array}$ & India & Urban \\
\hline $\begin{array}{l}\text { Fillenbaum } \\
1999\end{array}$ & $\begin{array}{l}\text { Everyday abilities scale for } \\
\text { India (EASI) }\end{array}$ & India & Rural \\
\hline Rao 2012 & $\begin{array}{lr}\text { National Institute of Mental } \\
\text { Health and } & \text { Neurosciences } \\
\text { (NIMHANS) } & \text { Headache } \\
\text { questionnaire } & \\
\end{array}$ & India & $\begin{array}{l}\text { Both urba } \\
\text { and rural }\end{array}$ \\
\hline Gaiki 2014 & $\begin{array}{l}\text { Mini nutritional assessment } \\
\text { (MNA) scale }\end{array}$ & India & Rural \\
\hline Pandav 2002 & $\begin{array}{l}\text { Functional Ability Scale - } \\
\text { EASI and HMSE }\end{array}$ & India & Rural \\
\hline Ghoshal 2015 & $\begin{array}{l}\text { Enhanced Asian Rome III } \\
\text { questionnaire (EAR3Q) }\end{array}$ & $\begin{array}{l}\text { India } \\
\text { (among } \\
\text { other Asian } \\
\text { countries) } \\
\end{array}$ & Both urbe \\
\hline Swati 2015 & $\begin{array}{l}\text { Dementia assessment by } \\
\text { Rapid test (DART) }\end{array}$ & India & $\begin{array}{l}\text { Both urba } \\
\text { and rural }\end{array}$ \\
\hline Umayal 2010 & $\begin{array}{l}\begin{array}{l}\text { Modified Bristol and Blessed } \\
\text { scale }\end{array} \\
\end{array}$ & Sri Lanka & $\begin{array}{l}\text { Both urban } \\
\text { and rural }\end{array}$ \\
\hline Diwan 2018 & Stroke impact scale & India & $\begin{array}{l}\text { Both urba } \\
\text { and rural }\end{array}$ \\
\hline & rssonal wellbeir & & Both $\mathrm{u}$ \\
\hline
\end{tabular}




\begin{tabular}{|l|l|l|l|l} 
& (PWI) & & and rural & \\
\hline Anjana 2015 & $\begin{array}{l}\text { MDRF- Physical Activity } \\
\text { Questionnaire (MPAQ) }\end{array}$ & $\begin{array}{l}\text { India urban } \\
\text { and rural }\end{array}$ & $>=2$ \\
\hline Cullati 2018 & Self-rated health (SRH) & India & $\begin{array}{l}\text { Both urban } \\
\text { and rural }\end{array}$ & $>=1$ \\
\hline Prasad 2018 & Modified Barthel index & India & Hospital & $18-\bar{\imath}$ \\
\hline Singh 2007 & $\begin{array}{l}\text { Activities of daily living } \\
\text { (ADL) IADL, modified } \\
\text { performance-oriented } \\
\text { mobility India } \\
\text { (POMA) assessment }\end{array}$ & Urban & $65-\varepsilon$ \\
\hline Gothwal 2013 & $\begin{array}{l}\text { Vision and Quality of Life } \\
\text { Index (VisQoL) }\end{array}$ & India & Hospital & 18 \\
yeal
\end{tabular}

All tools were either self-administered or administered through an investigator or health worker, except two tools, modified DSM-III checklist [19] and EASY-care [20], which were designed to be used by clinicians. The characteristics of the tools are provided in Table-2 below.

\begin{tabular}{|c|c|c|c|c|c|}
\hline \multicolumn{6}{|c|}{ Table-2 Characteristics of the Tools } \\
\hline Domain & Sub-domain & $\begin{array}{l}\text { Instrument } \\
\text { name }\end{array}$ & \begin{tabular}{|l|} 
No. \\
Items
\end{tabular} & of Language & $\begin{array}{l}\text { Durat } \\
\text { (mins }\end{array}$ \\
\hline & \multirow{4}{*}{ Diabetes } & MDRF-IDRS & 4 & $\begin{array}{l}\text { Not } \\
\text { specified }\end{array}$ & NA \\
\hline & & Simple DRS & 5 & NA & NA \\
\hline & & $\begin{array}{l}\text { DRS- urbar } \\
\text { Indians }\end{array}$ & 7 & NA & NA \\
\hline & & IDRS & 4 & NA & NA \\
\hline & $\overline{C V D}$ & EPOCH-2 & 13 & NA & NA \\
\hline & $\begin{array}{l}\text { Pulmonary } \\
\text { disease }\end{array}$ & SGRQ & 76 & Nepali & NA \\
\hline & Stroke & QVSFS & 8 & Urdu & 7 \\
\hline
\end{tabular}




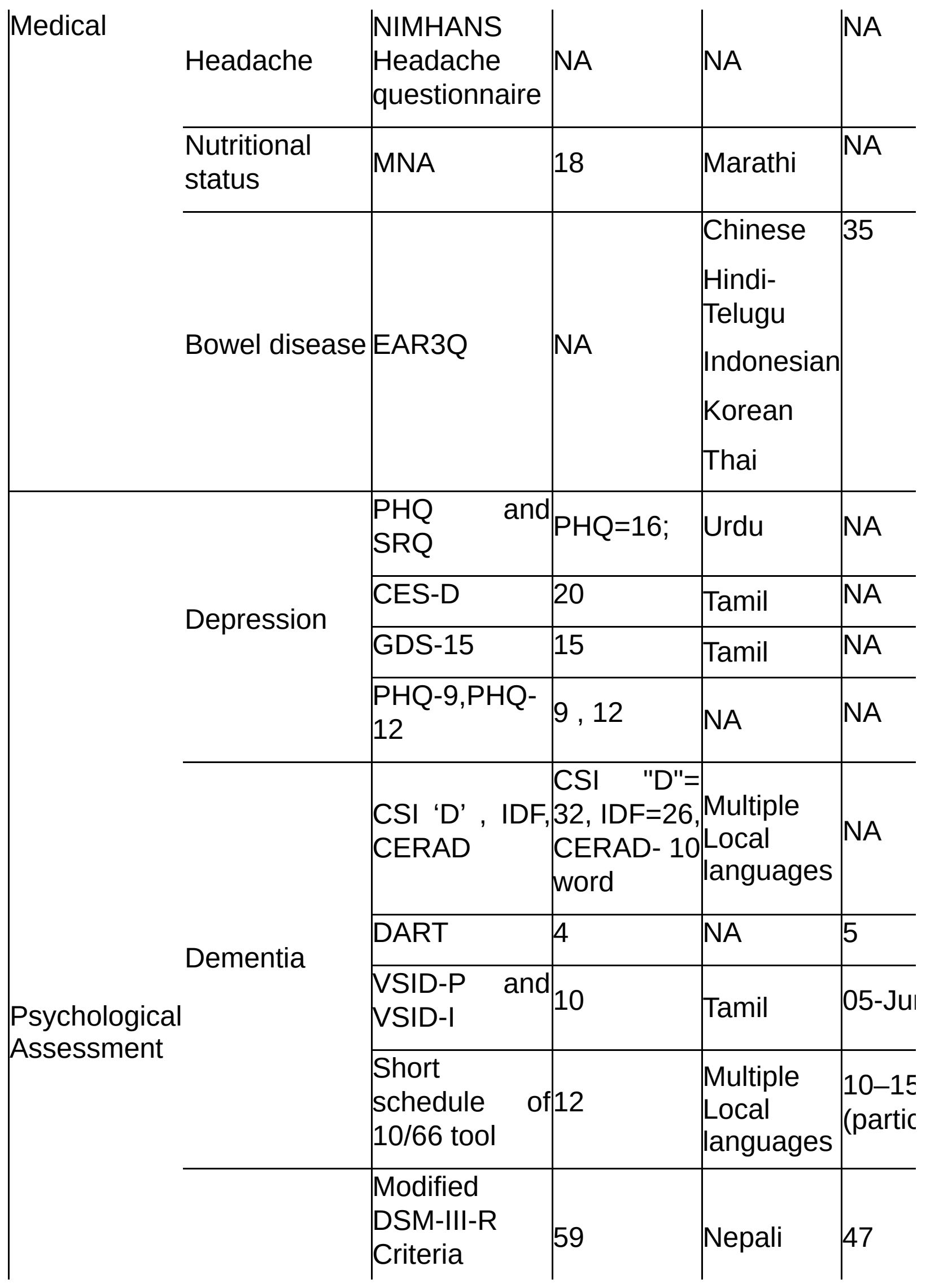




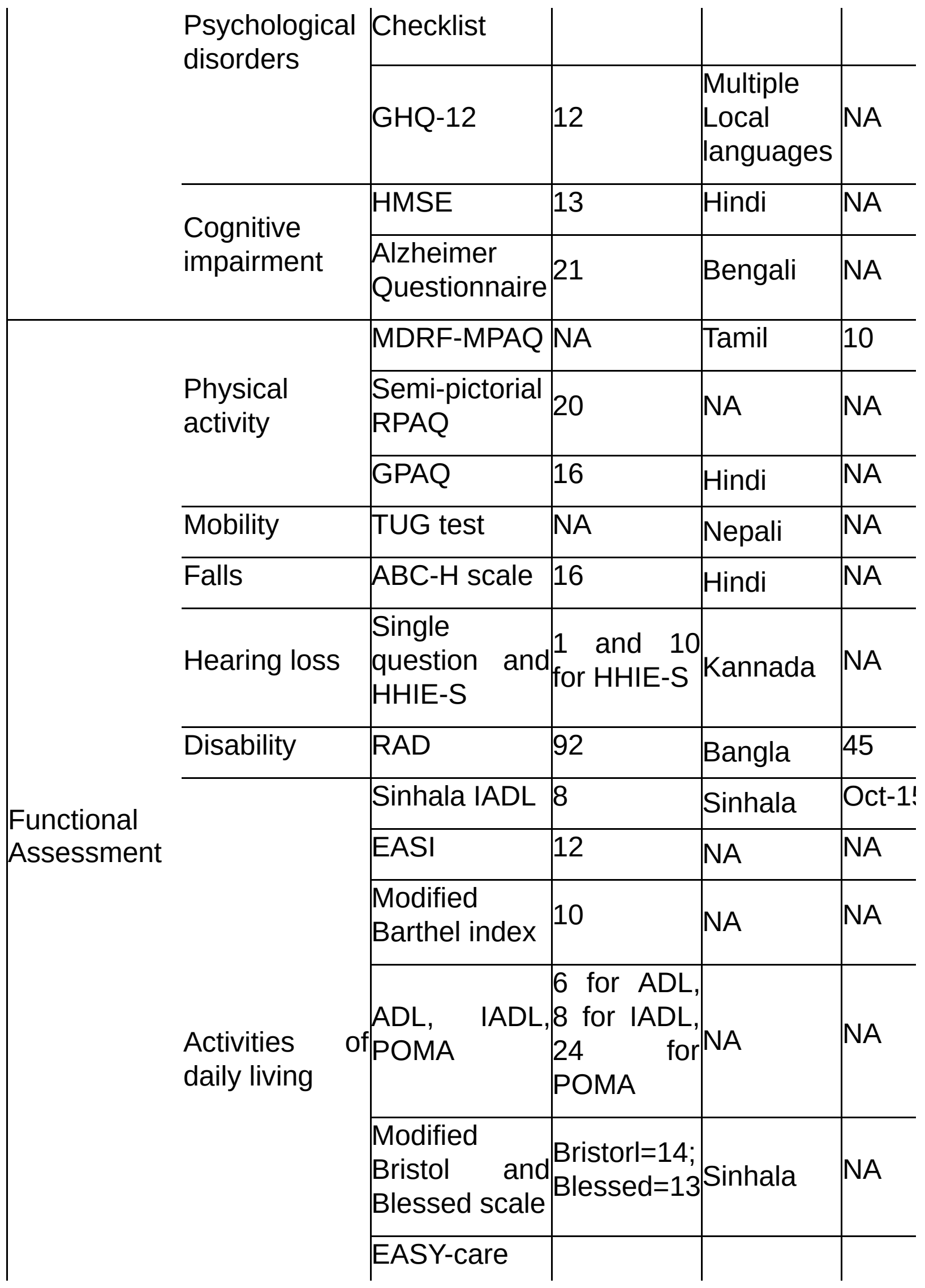




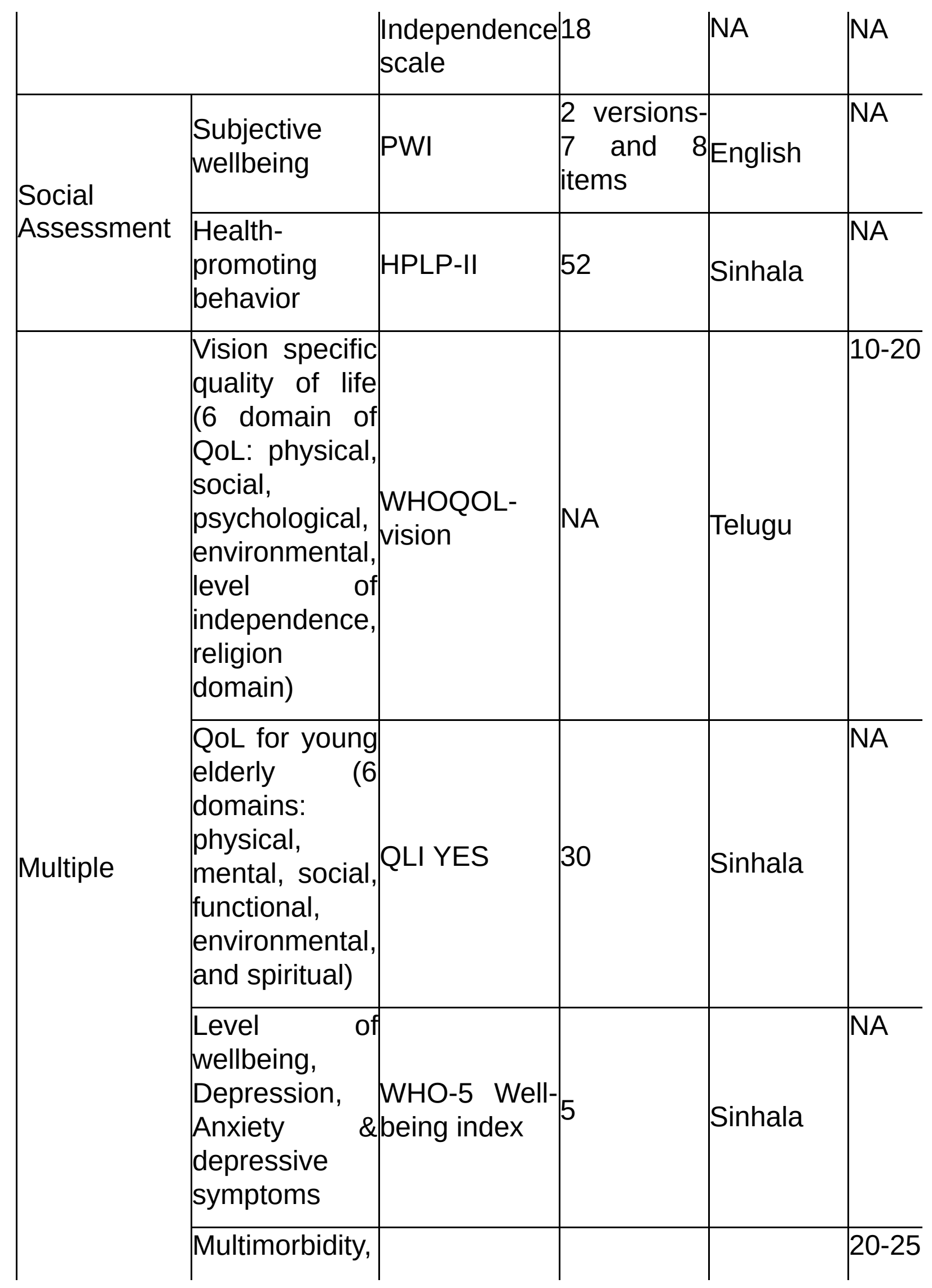




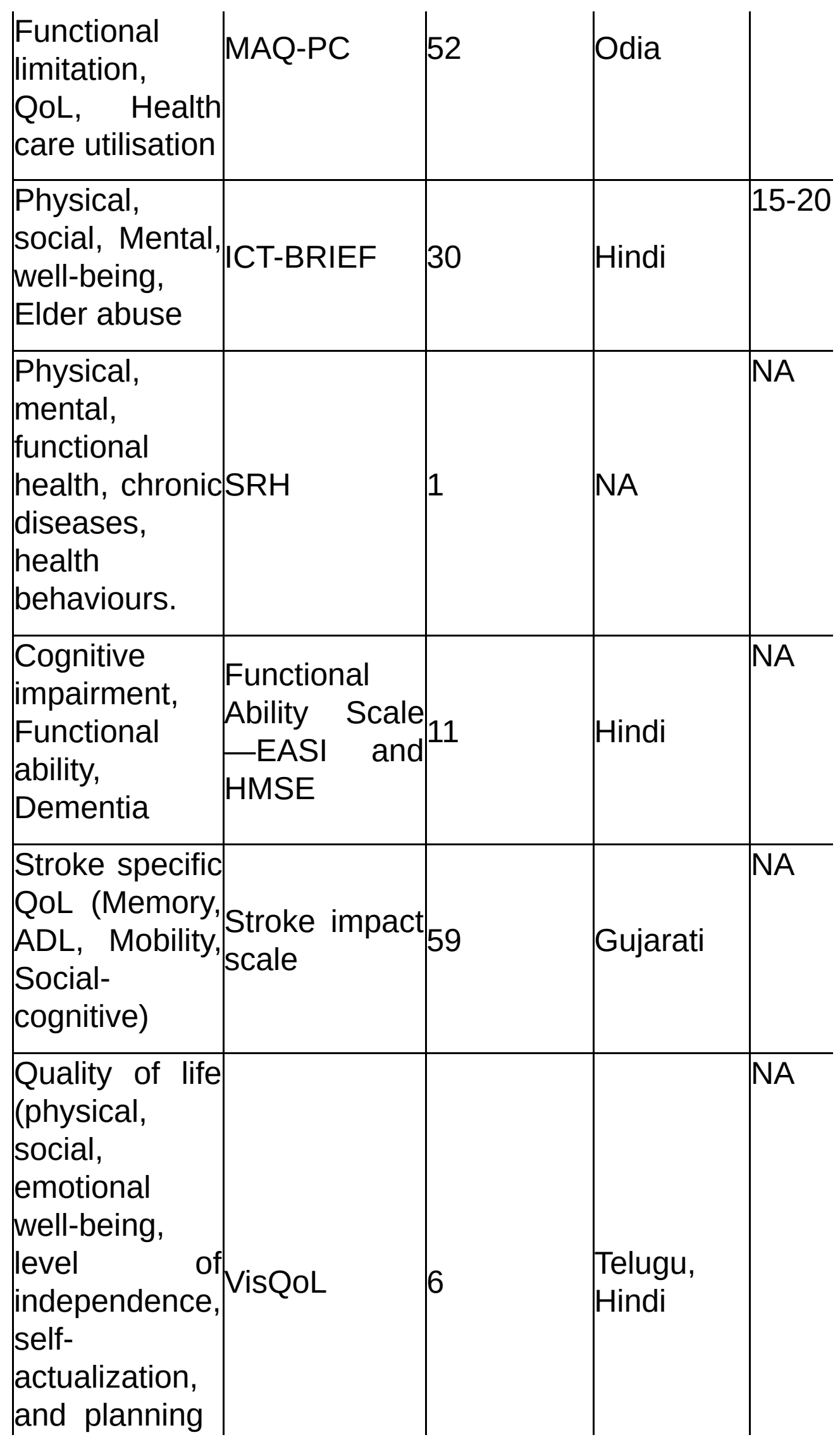


medRxiv preprint doi: https://doi.org/10.1101/2021.02.19.21252051; this version posted May 22, 2021. The copyright holder for this preprint (which was not certified by peer review) is the author/funder, who has granted medRxiv a license to display the preprint in perpetuity.

It is made available under a CC-BY 4.0 International license.

and

organization) 
medRxiv preprint doi: https://doi.org/10.1101/2021.02.19.21252051; this version posted May 22, 2021. The copyright holder for this preprint (which was not certified by peer review) is the author/funder, who has granted medRxiv a license to display the preprint in perpetuity.

\section{Measures of tools}

Majority of the tools were compared with a defined "Gold standard" to evaluate the sensitivity, specificity, predictive values, and cut-off optimization, which was reported in some form for 24 tools and summarized in table-3 below. The other common measures of tool design and validation reported were factorial analysis by Bartlett's test of sphericity, Kaiser-Meyer-Olkin (KMO) measure of sampling adequacy, exploratory and confirmatory factor analysis (EFA/CFA), content, convergent, divergent, criterion and construct validity, inter-item and spearman's correlation, reliability by tests of internal consistency such as Cronbach's $\alpha$ and test-retest reliability by intraclass correlation coefficients (ICCS), inter-rater reliability by Cohen's Kappa.

The available tools were categorized into five major domains: -

\section{A. Medical Assessment tools-}

The included studies on 10 tools related to medical assessment in older adults. Four tools - MDRF-IDRS[21], Simple DRS[22], DRSurban Indians[23], IDRS[24] were for risk scoring of diabetes which had showed moderate sensitivity and specificity.

The QVSFS[25]was intended to verify the stroke-free status, NIMHANS Headache questionnaire[26] for headache and EAR3Q[27] for bowel disease, all these tools had high sensitivity and NPV. The EPOCH-2[28] and MNA[29] were assessed cardiovascular and nutritional status respectively and reported moderate reliability for both. SGRQ[30] used to predict COPD and had high negative correlation between SGRQ scores and lung capacity.

B. Psychological assessment tools-

There were four studies each that assessed tools related to depression (PHQ/SRQ[31],CES-D[32], GDS-15[33], PHQ-9-12[34]) and dementia (CSI 'D'/IDF/CERAD[35], DART[36], VSID-P/VSIDI[37], Short schedule of 10/66 tool[38]). All of these reported moderates to high sensitivity and specificity as shown in table 3.

The DSM-III[19] checklist had moderate construct validity, variable reliability with poor alpha values for schizophrenia and mania, along 
medRxiv preprint doi: https://doi.org/10.1101/2021.02.19.21252051; this version posted May 22, 2021. The copyright holder for this preprint

(which was not certified by peer review) is the author/funder, who has granted medRxiv a license to display the preprint in perpetuity. It is made available under a CC-BY 4.0 International license .

with high alpha values for depression and anxiety. Whereas, the GHQ-12[39] tool showed moderate reliability. In case of HMSE[40] had moderate sensitivity and specificity, high NPV but poor PPV. However, Alzheimer's questionnaire[41] showed high sensitivity, specificity and high PPV.

\section{Functional assessment tools-}

There are five tools (Sinhala version of ADL[42], Modified Barthel index[43], EASI[44], EASY Care[20], modified Bristol and Blessed scales[45]) were validated for ADL assessment. Among them, Sinhala version of ADL[42] and EASY Care[20] independence scale had high reliability, Whereas Modified Barthel index[43], substantialhigh sensitivity and specificity, EASI[44] tool had shown moderate sensitivity and high specificity, with a poor PPV and the modified Bristol and Blessed scales[45] had very high sensitivity and moderate specificity.

RAD assesses disability with moderate sensitivity and high specificity. ABCH scale to assess falls had high sensitivity and specificity with a high PPV. Single question hearing handicap and HHIES both used for hearing loss, had poor sensitivity and high specificity with poor NPV.

D. Social assessment tools-

Subjective wellbeing as assessed by PWI[46] and health-promoting behavior assessed by HPLP-II[18]. Both tools had high reliability.

E. Multiple domain assessment tools-

A total of nine tools assessed more than one domain and all had some form of a quality-of-life assessment included in them.

The WHOQOL vision[47], VisQoL[48], The QLI-YES[49] included 6 different domains of quality of life and reported high to moderate reliability.

The WHO-5 well-being index[50] is used to assess and compare depression, anxiety \& depressive symptoms. The translated version demonstrated good content and face validity with high reliability.

The MAQ-PC[51] tool assessed multimorbidity, functional limitation, 
medRxiv preprint doi: https://doi.org/10.1101/2021.02.19.21252051; this version posted May 22, 2021. The copyright holder for this preprint (which was not certified by peer review) is the author/funder, who has granted medRxiv a license to display the preprint in perpetuity.

depression, health-related quality of life and health care utilization. It had an overall moderate to high reliability and validity. ICT-BRIEF tool[52] and $\mathrm{SRH}[53]$ tools to assess different domains of quality of life had high reliability and t moderate validity respectively

Stroke impact scale[54] assessed the stroke-specific QoL and reported moderate positive correlations between constructs of memory, communication, ADL, mobility, and hand function. It showed weak positive correlation with participation and physical domains.

The psychometric properties of the tools described above are summarized in Table-3 and also available in supplementary file (Appendix-3).

\begin{tabular}{|c|c|c|c|c|c|c|}
\hline \multicolumn{7}{|c|}{ Table-3 psychometric properties of the Tools } \\
\hline Domain & Tool name & Cut of & $\begin{array}{l}\text { Sensitiv } \\
(\%) \quad(95 \\
\text { Cl) }\end{array}$ & $\begin{array}{l}\text { Specificity } \\
(\%)\end{array}$ & PPV (\%) & $\begin{array}{l}\text { NPV } \\
(\%)\end{array}$ \\
\hline \multirow{7}{*}{ Medical } & MDRF-IDRS & $\geq 60$ & 62.2 & 73.7 & 19.7 & 94.9 \\
\hline & Simple DRS & $>9$ & $\begin{array}{l}75.7 \\
(62.1- \\
85.5)\end{array}$ & $\begin{array}{l}61.6 \\
(58.1- \\
65.0)\end{array}$ & $\begin{array}{l}13.1 \\
(9.7- \\
17.2) \\
\end{array}$ & $\begin{array}{l}97.0 \\
(95 . C \\
98.3 \\
\end{array}$ \\
\hline & QVSFS & & $\begin{array}{l}77.1 \\
(64.1- \\
86.9)\end{array}$ & $\begin{array}{l}85.8 \\
(83.5- \\
87.5)\end{array}$ & $\begin{array}{l}48.7 \\
(40.5- \\
54.9)\end{array}$ & $\begin{array}{l}95.5 \\
(93.6 \\
97.4 \\
\end{array}$ \\
\hline & $\begin{array}{l}\text { DRS-Urban } \\
\text { Indians }\end{array}$ & & 76.6 & 59.9 & 9.4 & 97.9 \\
\hline & IDRS & $\geq 60$ & 72.5 & 60.1 & 17.0 & 95.1 \\
\hline & $\begin{array}{l}\text { NIMHANS } \\
\text { Headache }\end{array}$ & & $\begin{array}{l}88 \\
(83-91)\end{array}$ & $\begin{array}{l}81 \\
(74-87)\end{array}$ & $\begin{array}{l}89 \\
(84-92)\end{array}$ & $\begin{array}{l}80 \\
(73- \\
86) \\
\end{array}$ \\
\hline & EAR3Q & & $>60$ & $>60$ & & \\
\hline & $\mathrm{PHQ}$ & $5 / 6$ & 69.6 & 85.2 & 78.9 & 77.8 \\
\hline
\end{tabular}




\begin{tabular}{|c|c|c|c|c|c|c|}
\hline & SRQ & $5 / 6$ & 93.1 & 80.8 & 77.9 & 94.1 \\
\hline & PHQ-12 & $\geq 4$ & 92.0 & 90.7 & 76.7 & 97.1 \\
\hline & \begin{tabular}{|l} 
Short \\
schedule of \\
$10 / 66$ tool \\
\end{tabular} & & 94.2 & $80.2 \%$ & & \\
\hline & VSID-P & & 66.7 & 77.6 & 8.3 & 98.7 \\
\hline Psychological & VSID-I & & 100 & 79.2 & 13.0 & 100 \\
\hline & DART & & 95.5 & 60.0 & 70.2 & 93.0 \\
\hline & GDS-15 & & 80.0 & 47.6 & & \\
\hline & HMSE & & 81.3 & 60.2 & 9.8 & 98.4 \\
\hline & $\begin{array}{l}\text { Alzheimer's } \\
\text { questionnaire }\end{array}$ & & $\begin{array}{l}85.7 \% \\
(95 \% \\
\mathrm{Cl}=74.2- \\
92.6)\end{array}$ & \begin{tabular}{|l}
$96.4 \%$ \\
$(95 \%$ \\
$\mathrm{Cl}=89.9-$ \\
$98.7)$
\end{tabular} & $\begin{array}{l}94.1 \% \\
(95 \% \\
C l=84.0- \\
97.9 \%) .\end{array}$ & \\
\hline & RAD & & 62.4 & 81.2 & & \\
\hline & ABC-H scale & $>58.13$ & $\begin{array}{l}86.3 \\
(65.1- \\
97.1) \\
\end{array}$ & $\begin{array}{l}87.3 \\
(79.4- \\
93.1) \\
\end{array}$ & $\begin{array}{l}59.4 \\
(40.6- \\
76.3) \\
\end{array}$ & $\begin{array}{l}96.8^{\prime} \\
(90 . \varepsilon \\
99.3^{\prime}\end{array}$ \\
\hline & $\begin{array}{l}\text { Modified } \\
\text { Bristol Scale }\end{array}$ & & 100.0 & 74.2 & & \\
\hline & $\begin{array}{l}\text { Modified } \\
\text { Blessed } \\
\text { scale }\end{array}$ & & 100.0 & 71.0 & & \\
\hline & $\begin{array}{l}\text { Single } \\
\text { question } \\
\text { hearing }\end{array}$ & $>25 \mathrm{dl}$ & 30.9 & 93.9 & 92.9 & 34.6 \\
\hline
\end{tabular}




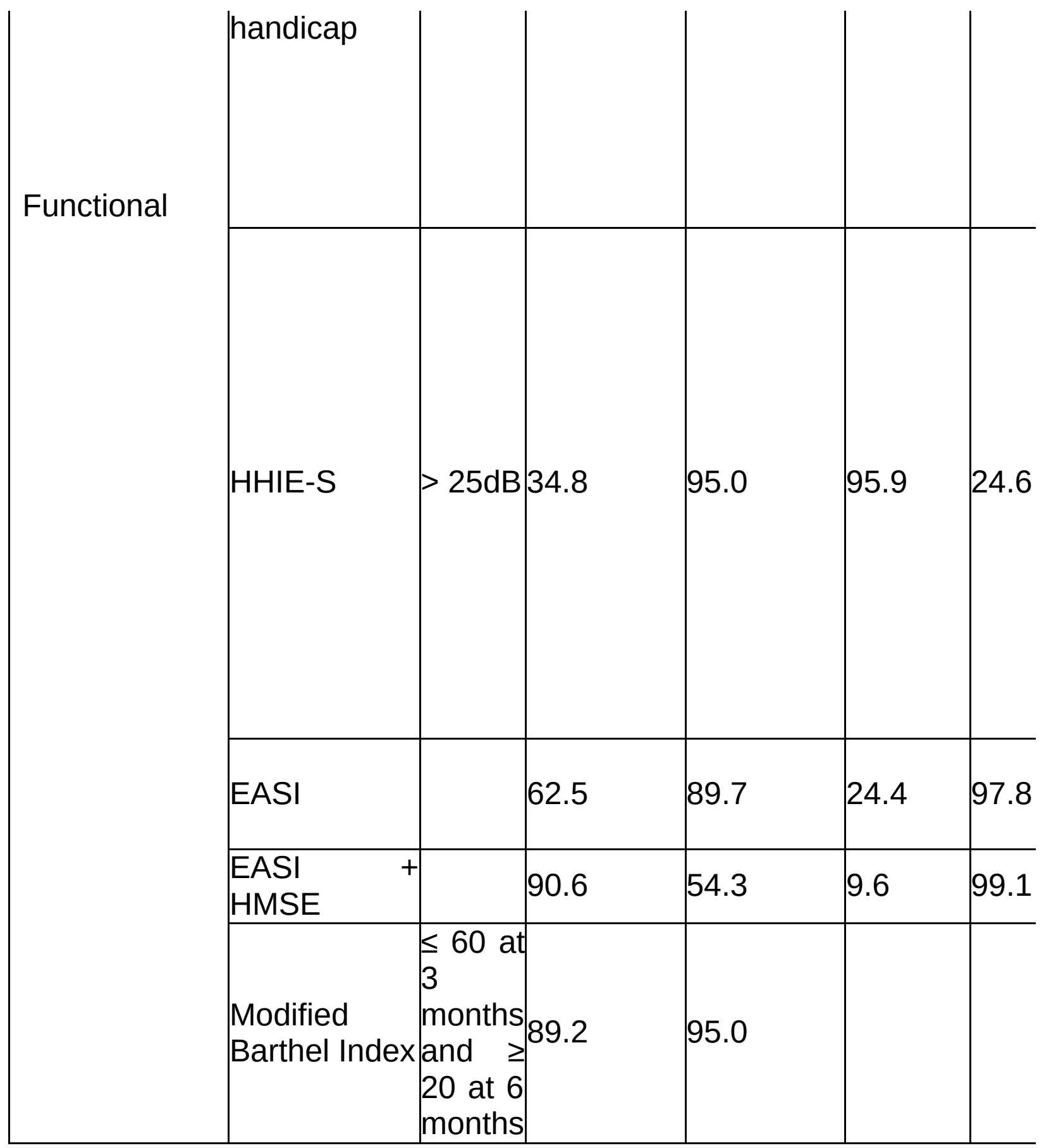




\section{Discussion}

This systematic scoping review aimed to describe available tools to assess geriatric health in community settings in South Asia. We found that there is a paucity of tools that can be used to evaluate elderly health comprehensively. Majority of tools available evaluate specific health outcomes, rather than broader conceptualizations of complete wellbeing and hence are not suitable for use in assessment of multiple domains of health. A large majority(29 instruments) were adopted from pre-existing instruments, mostly developed for the western population. This indicates insufficient research and uptake of health measurements and scales particularly for the South Asian population. While two studies were from before 2000, many studies (32) were published in the past decade. Research aimed at tool development and validation in South Asia is probably a relatively recent phenomenon.

A combination of steady declining birth rate and increasing life expectancy has been seen in South Asia that has resulted in an increasing aging population, along with an increasing burden of morbidity. This amplifies the need for CGA and the tools necessary for its implementation. , This would play an important role to decide individual needs and thus provide person-centered care. This review also reported medical and functional health assessment seems to be the major domains of CGA represented by maximum number of tools rather than the whole spectrum of health. While assessing geriatric QoL, environment plays an essential role, but tools to assess this domain are the least frequently found. Studies conducted on elderly population in China have shown a clear association between environmental factors 
medRxiv preprint doi: https://doi.org/10.1101/2021.02.19.21252051; this version posted May 22, 2021. The copyright holder for this preprint (which was not certified by peer review) is the author/funder, who has granted medRxiv a license to display the preprint in perpetuity. It is made available under a CC-BY 4.0 International license .

and survival, where air pollution was shown to have a significant association with elderly health[55]. Similarly, tools assessing financial burden of illness, access to health care or social health were not found.

The priority in the region has traditionally been on addressing infectious diseases, maternal and child health challenges. Elderly health has therefore remained a neglected area of policy, practice and subsequently research in the region. Although few targeted health programs such as the "National Programme for Health Care of the Elderly (NPHCE)" in India [56] have been initiated in recent years, their impact is yet to be evaluated. These programs envision geriatric assessment and screening in their activities but the uptake has been slow due to unavailability of tools for the same that can be used by the community health workers.

Most of the programs in developing countries have attached less importance to home-based elderly care because of the emphasis given to institutional, mostly medical care. This finding is supported by our review where only one tool MAQ-PC[51] included most of the domains (Medical, Psychological, Functional) of CGA but that too considering the institutional-based care needs of the participants.

Health systems need to appreciate and be prepared for the shift from communicable to non-communicable diseases in the coming years. An increasing focus on healthcare for the elderly in the coming decades is inevitable. Tools for the health assessment and screening of this group need to be contextually developed and validated in multiple settings to realize the goals of the national programs.

\section{Limitation of the review}

Only peer-reviewed articles published in English language were selected, which may introduce selection bias, however, it is also important to note that the primary language for scientific communication in the whole of South Asia is English. We have not evaluated the quality of the studies included. We have limited our studies to South Asia, this could increase the chance of missing out the studies conducted in other LMICs. However, the compliance of the review with PRISMA guidelines with robust and systematic methodology strengthens the confidence in findings. 


\section{Conclusion}

This review indicated a significant gap in tools available for CGA in the South Asian population that can be used in community settings. No tool combining all aspects of CGA has been reported from the region. Acknowledging the significance of context-specific robust health assessment tools, there is a need for culturally sensitive and practical tools that can be used to assess geriatric health.

\section{Authors' Contributions}

SP1 \& TB were equally contributed to the development of search strategy, screening, hand searching, charting, and analysis of the data. SP1 drafted the article. JSK was responsible for conceptualization, manuscript review, and editing.SP2 was responsible for manuscript review and editing. All the authors have read and agreed to the published version of manuscript.

\section{Funding}

This review received no external funding.

\section{Conflict of Interest}

The authors declare no conflict of interest

\section{References}

1. Organization world health. The global burden of disease 2004. Updat World Heal Organ 2004:146.

2. Prince MJ, Wu F, Guo Y et al. The burden of disease in older people and implications for health policy and practice. Lancet 2015;385:549-62.

3. Alemayehu B, Warner KE. The lifetime distribution of health care costs. Health Serv Res 2004;39:627-42.

4. Kowal $\mathrm{P}, \mathrm{Kahn} \mathrm{K}, \mathrm{Ng} \mathrm{N}$ et al. Ageing and adult health status in eight 
medRxiv preprint doi: https://doi.org/10.1101/2021.02.19.21252051; this version posted May 22, 2021. The copyright holder for this preprint (which was not certified by peer review) is the author/funder, who has granted medRxiv a license to display the preprint in perpetuity. It is made available under a CC-BY 4.0 International license .

lower-income countries: The INDEPTH WHO-SAGE collaboration. Glob Health Action 2010;3:11-22.

5. Jiang S, Li P. Current development in elderly comprehensive assessment and research methods. Biomed Res Int 2016;2016, DOI: $10.1155 / 2016 / 3528248$.

6. Zagonel V. Importance of a comprehensive geriatric assessment in older cancer patients. Eur J Cancer 2001;37, DOI: 10.1016/s09598049(01)80024-1.

7. Nord M, Östgren CJ, Marcusson $\mathrm{J}$ et al. Staff experiences of a new tool for comprehensive geriatric assessment in primary care (PASTEL): a focus group study: Primary care staff experiences of geriatric assessment. Scand J Prim Health Care 2020;38:132-45.

8. Solomon D, Brown AS, Brummel-Smith $\mathrm{K}$ et al. National Institutes of Health Consensus Development Conference Statement: Geriatric assessment methods for clinical decision-making. J Am Geriatr Soc 1988;36:342-7.

9. University of Marylan. GERIATRIC ASSESSMENT TOOLS.

10. Katz S, Akpom CA. A measure of primary sociobiological functions. Int J Heal Serv 1976;6:493-508.

11. Lawton MP, Moss M, Fulcomer $\mathrm{M}$ et al. A research and service oriented multilevel assessment instrument. Journals Gerontol 1982;37:91-9.

12. Thompson T. Immigrant Populations. Encycl Heal Commun 2014:19.

13. Vrabel M. Preferred reporting items for systematic reviews and metaanalyses. Oncol Nurs Forum 2015;42:552-4.

14. Arksey H, O'Malley L. Scoping studies: Towards a methodological framework. Int J Soc Res Methodol Theory Pract 2005;8:19-32.

15. Peters MDJ, Godfrey CM, Khalil H et al. Guidance for conducting systematic scoping reviews. Int J Evid Based Healthc 2015;13:141-6.

16. Dr. Jaya Singh Kshatri. Comprehensive Geriatric Assessment (CGA) - time for knowledge translation.

17. Wieland D, Hirth V. Comprehensive Geriatric Assessment. Cancer 
medRxiv preprint doi: https://doi.org/10.1101/2021.02.19.21252051; this version posted May 22, 2021. The copyright holder for this preprint

(which was not certified by peer review) is the author/funder, who has granted medRxiv a license to display the preprint in perpetuity.

Control 2003;10:454-62.

18. Rathnayake N, Alwis G, Lenora J et al. Applicability of health promoting lifestyle profile-Il for postmenopausal women in Sri Lanka; A validation study. Health Qual Life Outcomes 2020;18:122.

19. Tausig M, Subedi S, Broughton CL et al. Measuring community mental health in developing societies: Evaluation of a checklist format in Nepal. Int J Soc Psychiatry 2003;49:269-83.

20. Jotheeswaran AT, Dias A, Philp I et al. Calibrating EASY-Care independence scale to improve accuracy. Age Ageing 2016;45:890-3.

21. Adhikari P, Pathak R, Kotian S. Validation of the MDRF - Indian diabetes risk score (IDRS) in another South Indian population through the boloor diabetes study (BDS). J Assoc Physicians India 2010;58:434-6.

22. Bhowmik B, Akhter A, Ali L et al. Simple risk score to detect rural Asian Indian (Bangladeshi) adults at high risk for type 2 diabetes. $J$ Diabetes Investig 2015;6:670-7.

23. Ramachandran A, Snehalatha C, Vijay V et al. Derivation and validation of diabetes risk score for urban Asian Indians. Diabetes Res Clin Pract 2005;70:63-70.

24. Mohan V, Deepa R, Deepa M et al. A simplified Indian Diabetes Risk Score for screening for undiagnosed diabetic subjects. $J$ Assoc Physicians India 2005;53:759-63.

25. Khan M, Kamal AK, Islam $M$ et al. Can Trained Field Community Workers Identify Stroke Using a Stroke Symptom Questionnaire as well as Neurologists? J Stroke Cerebrovasc Dis 2015;24:91-9.

26. Rao GN, Kulkarni GB, Gururaj $G$ et al. The burden of headache disorders in India: Methodology and questionnaire validation for a community-based survey in Karnataka State. $J$ Headache Pain 2012;13:543-50.

27. Ghoshal UC, Gwee KA, Chen M et al. Development, translation and validation of Enhanced Asian Rome III questionnaires for diagnosis of functional bowel diseases in major Asian languages: A Rome foundationAsian neurogastroenterology and motility association working team report. J Neurogastroenterol Motil 2015;21:83-92.

28. Corsi DJ, Subramanian S V., McKee M et al. Environmental Profile of 
medRxiv preprint doi: https://doi.org/10.1101/2021.02.19.21252051; this version posted May 22, 2021. The copyright holder for this preprint (which was not certified by peer review) is the author/funder, who has granted medRxiv a license to display the preprint in perpetuity. It is made available under a CC-BY 4.0 International license .

a Community's Health (EPOCH): An Ecometric Assessment of Measures of the Community Environment Based on Individual Perception. PLoS One 2012;7:e44410.

29. Gaiki V, Wagh V. Reliability of Mini-Nutritional Assessment Scale in Rural Setup of a Tertiary Health Care Hospital in Central India. 2014;2.

30. Sherpa CT, LeClerq SL, Singh S et al. Validation of the St. George's Respiratory Questionnaire in Nepal. Chronic Obstr Pulm Dis J COPD Found 2015;2:281-9.

31. Husain N, Gater R, Tomenson B et al. Comparison of the personal health questionnaire and the self reporting questionnaire in rural Pakistan. J Pak Med Assoc 2006;56:366-70.

32. Chokkanathan S, Mohanty J. Factor structure of the CES-D scale among older adults in Chennai, India. Aging Ment Heal 2013;17:517-25.

33. Sarkar S, Kattimani S, Roy G et al. Validation of the Tamil version of short form Geriatric Depression Scale-15. J Neurosci Rural Pract 2015;6:442-6.

34. Poongothai S, Pradeepa R, Ganesan A et al. Reliability and validity of a modified PHQ-9 item inventory (PHQ-12) as a screening instrument for assessing depression in Asian Indians (CURES-65). J Assoc Physicians India 2009;57.

35. Prince $\mathrm{M}$, Acosta $\mathrm{D}$, Chiu $\mathrm{H}$ et al. Dementia diagnosis in developing countries: A cross-cultural validation study. Lancet 2003;361:909-17.

36. B S, V S. Dementia Assessment by Rapid Test (DART): An Indian Screening T ool for Dementia. J Alzheimer's Dis Park 2015;05, DOI: 10.4172/2161-0460.1000198.

37. Stanley R, Kuruvilla A, Kumar $S$ et al. The Vellore screening instruments and strategies for the diagnosis of dementia in the community. Int Psychogeriatrics 2009;21:539-47.

38. Stewart $R$, Guerchet $M$, Prince $M$. Development of a brief assessment and algorithm for ascertaining dementia in low-income and middle-income countries: The 10/66 short dementia diagnostic schedule. BMJ Open 2016;6:e010712.

39. Qin M, Vlachantoni A, Evandrou $M$ et al. General Health Questionnaire-12 reliability, factor structure, and external validity among 
medRxiv preprint doi: https://doi.org/10.1101/2021.02.19.21252051; this version posted May 22, 2021. The copyright holder for this preprint (which was not certified by peer review) is the author/funder, who has granted medRxiv a license to display the preprint in perpetuity. It is made available under a CC-BY 4.0 International license .

older adults in India. Indian J Psychiatry 2018;60:56-9.

40. Ganguli M, Ratcliff G, Chandra $V$ et al. A hindi version of the MMSE: The development of a cognitive screening instrument for a largely illiterate rural elderly population in india. Int $\mathrm{J}$ Geriatr Psychiatry 1995;10:367-77.

41. Dasgupta A, Biswas A, Paul B et al. Comparative Analysis of Alzheimer Questionnaire and Montreal Cognitive Assessment Tool for Cognitive Impairment Screening among the Elderly Population. J Clin Diagnostic Res 2020;14:5-9.

42. Siriwardhana DD, Walters K, Rait G et al. Cross-cultural adaptation and psychometric evaluation of the Sinhala version of lawton instrumental activities of daily living scale. PLoS One 2018;13:e0199820.

43. Prasad K, Kumar A, Misra S et al. Reliability and validity of telephonic Barthel Index: an experience from multi-centric randomized control study. Acta Neurol Belg 2018;118:53-9.

44. Fillenbaum GG, Chandra V, Ganguli M et al. Development of an activities of daily living scale to screen for dementia in an illiterate rural older population in India. Age Ageing 1999;28:161-8.

45. Umayal $S$, Kulathunga $M$, Somaratne $S$ et al. Validation of a functional screening instrument for dementia in an elderly sri lankan population: Comparison of modified bristol and blessed activities of daily living scales. BMC Res Notes 2010;3:0-4.

46. McIntyre E, Saliba A, McKenzie K. Subjective wellbeing in the Indian general population: a validation study of the Personal Wellbeing Index. Qual Life Res 2020;29:1073-81.

47. Dandona R, Dandona L, McCarty CA et al. Adaptation of WHOQOL as health-related quality of life instrument to develop a vision-specific instrument. Indian J Ophthalmol 2000;48:65-70.

48. Gothwal VK, Bagga DK. Vision and quality of life index: Validation of the Indian version using rasch analysis. Investig Ophthalmol Vis Sci 2013;54:4871-81.

49. De Silva SHP, Jayasuriya AR, Rajapaksa LC et al. Development and Validation of a Measure of Quality of Life for the Young Elderly in Sri Lanka. Bland Bowling, Cronin, De Beni, Emery, Fayers, Guyatt, Hattie, 
Hodkinson, Huijer, Ingersoll-Dayton, Juniper, Kelley-Gillespie, Ku, Kumarapeli, Ledesma, Mahoney, McDowell, Nilsson, Osborne, Paschoal, Portney, Power, Rockwood, Shrout, Thanakwang, Wands, Yo B (ed.). Asia-Pacific J Public Heal 2016;28:115S-125S.

50. Perera BPR, Jayasuriya R, Caldera A et al. Assessing mental wellbeing in a Sinhala speaking Sri Lankan population: Validation of the WHO-5 well-being index. Health Qual Life Outcomes 2020;18:305.

51. Pati S, Hussain MA, Swain S et al. Development and Validation of a Questionnaire to Assess Multimorbidity in Primary Care: An Indian Experience. Biomed Res Int 2016;2016, DOI: 10.1155/2016/6582487.

52. Chatterjee P, Rebok GW, Dwivedi SN et al. Development of integrated care tool - BRIEF for screening the unmet psychosociomedical needs of older Indians. Indian J Public Health 2019;63:51-7.

53. Cullati S, Mukhopadhyay S, Sieber $\mathrm{S}$ et al. Is the single self-rated health item reliable in India? A construct validity study. BMJ Glob Heal 2018;3:1-12.

54. Diwan. 1/22/2021 Article Fulle Text. 2021;47:1-8.

55. Zeng $\mathrm{Y}, \mathrm{Gu} \mathrm{D}$, Purser $\mathrm{J}$ et al. Associations of environmental factors with elderly health and mortality in china. Am $\mathrm{J}$ Public Health 2010;100:298-305.

56. Verma R, Khanna P. National program of health-care for the elderly in India: A hope for healthy ageing. Int J Prev Med 2013;4:1103-7. 
medRxiv preprint doi: https://doi.org/10.1101/2021.02.19.21252051; this version posted May 22, 2021. The copyright holder for this preprint (which was not certified by peer review) is the author/funder, who has granted medRxiv a license to display the preprint in perpetuity.

It is made available under a CC-BY 4.0 International license. 